\title{
Successful use of radiofrequency ablation for the management of a recurrent ampullary adenoma with intraductal extension
}

Bharat Rao, MD, Mrinal Garg, MD, Shailendra Singh, MD, Abhishek Gulati, MD, Shyam Thakkar, MD

We present a case in which radiofrequency ablation (RFA) was used for the treatment of an ampullary adenoma with persistent intraductal growth despite prior treatments with endoscopic ampullectomy and argon plasma coagulation (APC). Cholangioscopy was used to evaluate the full extent of intraductal tissue. RFA was applied over 2 sessions (7-10 watts) for 90 seconds with the achievement of successful ablation (Video 1, available online at www. VideoGIE.org). There was no endoscopic or histologic evidence of adenoma recurrence at a 6-month surveillance visit.

Endoscopic management of advanced ampullary adenomas affords patients an option when they are not surgical candidates. However, tumors with intraductal growth are challenging to definitively treat endoscopically. APC is commonly used to ablate intraductal tissue, but recurrence is not uncommon despite its use. RFA is perhaps a more effective available modality. Initial studies have demonstrated the feasibility and effectiveness of RFA alone or in combination with other modal-

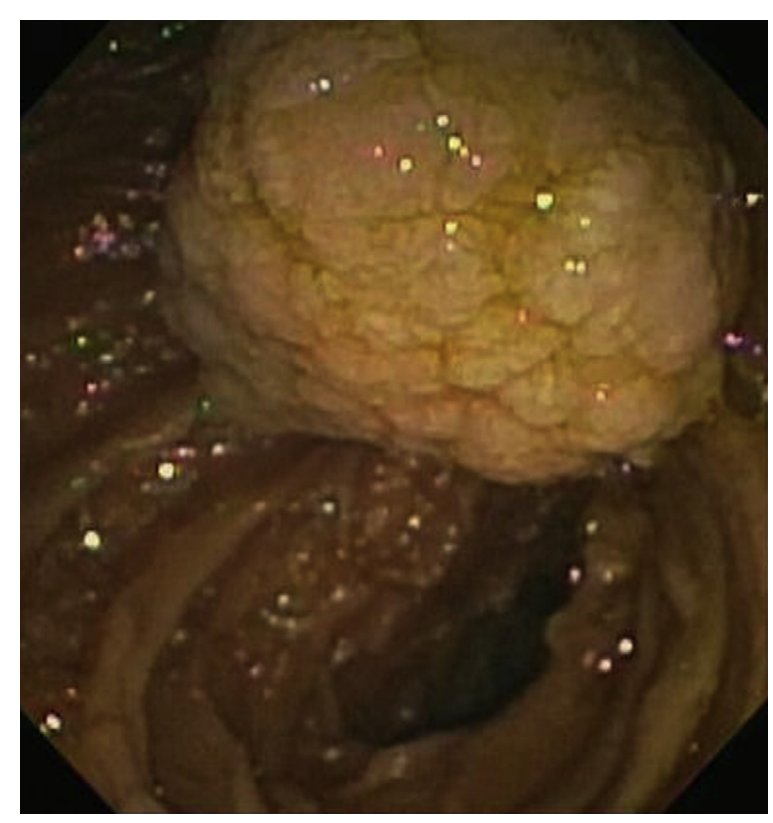

Figure 1. Large, 4-cm tubulovillous adenoma arising from the major duodenal papilla. ities in successfully treating intraductal extension. The procedure carries an adverse risk of stricture formation, and prophylatic biliary stents are commonly placed. ${ }^{1-3}$

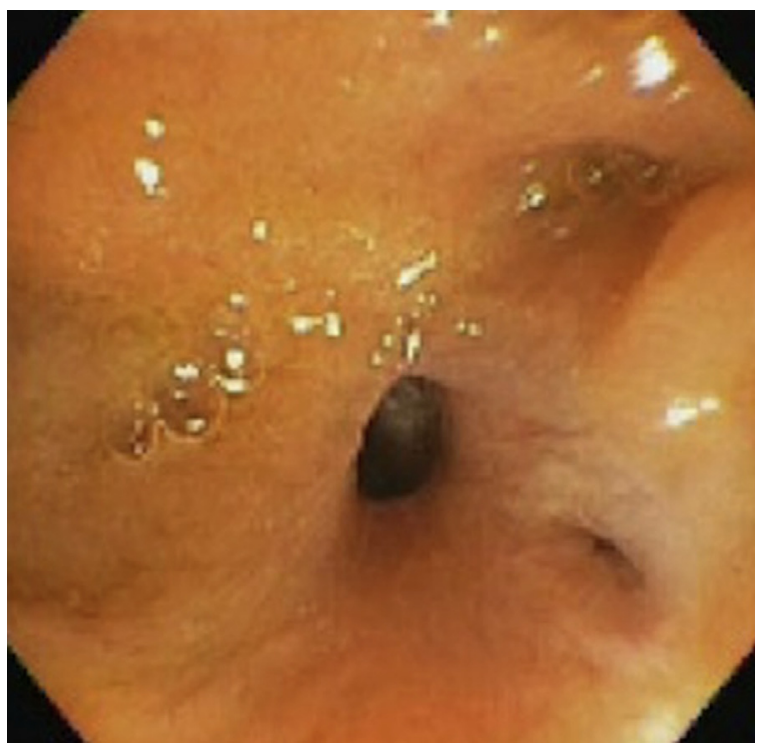

Figure 2. Endoscopic appearance of disease resolution after piecemeal resection and treatments with argon plasma coagulation.

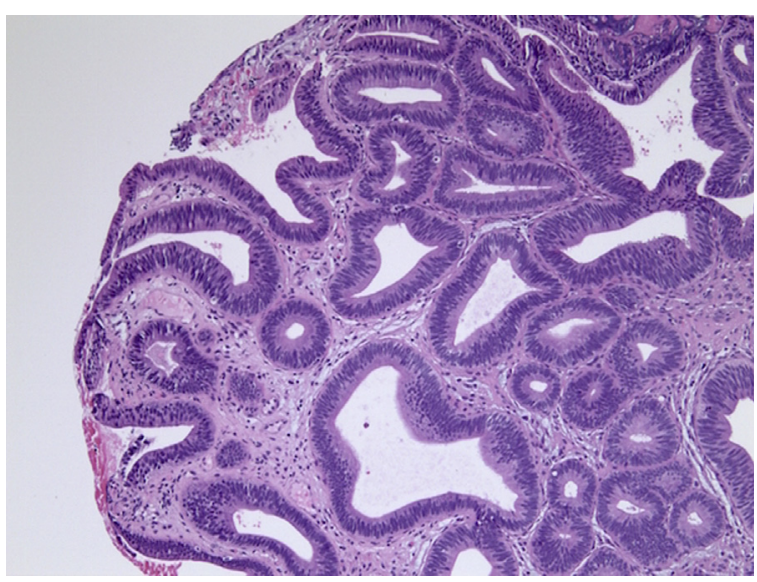

Figure 3. Biopsy specimen obtained from the distal common bile duct after ablation with argon plasma coagulation, showing persistent tubulovillous adenoma (H\&E, orig. mag. $\times 10$ ).

Written transcript of the video audio is available online at www.VideoGIE.org. 


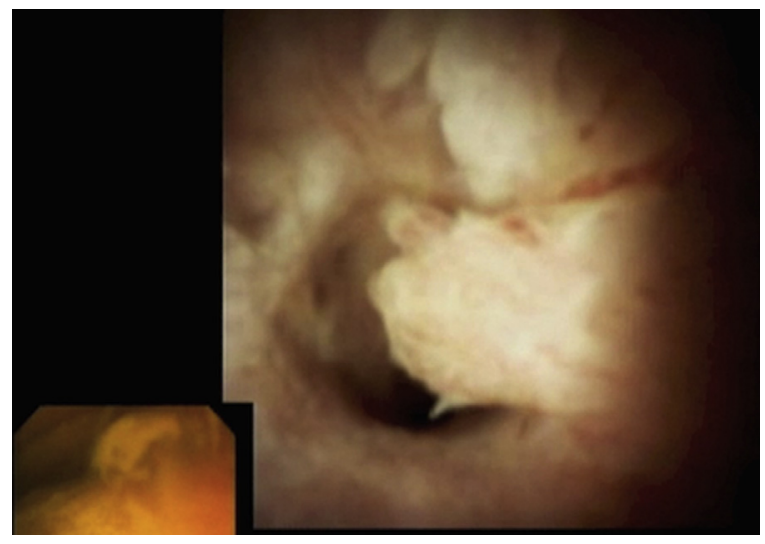

Figure 4. Polyp visualized at the 3 o'clock position extending circumferentially in the distal common bile duct.

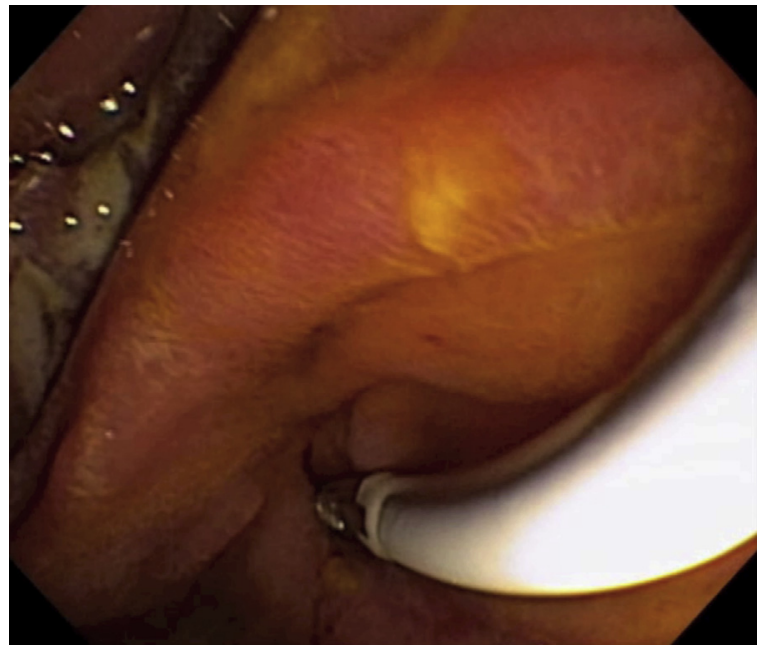

Figure 5. An $8 \mathrm{~F}$ probe with a $2.5-\mathrm{cm}$ ablation zone positioned over a wire into the distal biliary tree to perform radiofrequency ablation.

We further the understanding of RFA for intraductal extension by describing our experience of its use in a patient who continued to have recurrence despite previous treatments.

A 71-year-old woman had a history of a large, 4-cm, tubulovillous ampullary adenoma (Fig. 1). EUS, initially used for staging, did not show invasion into the duodenal wall nor infiltration of the mass into the pancreatic duct (PD) or common bile duct (CBD)Perihepatic and pancreatic lymph nodes were seen, and FNA results were unremarkable for tumor cells. CT revealed diffuse thickening of the extrahepatic bile duct extending from the ampulla without a discrete mass. Endoscopic resection was performed. Pathologic examination of the specimen on initial resection showed focal high-grade dysplasia (focal low-grade dysplasia was seen extending to the resection margin). The patient had a history of primary sclerosing cholangitis with cirrhosis and was not medically suitable to undergo surgical resection. Initial endoscopic management consisted of piecemeal polypectomy,

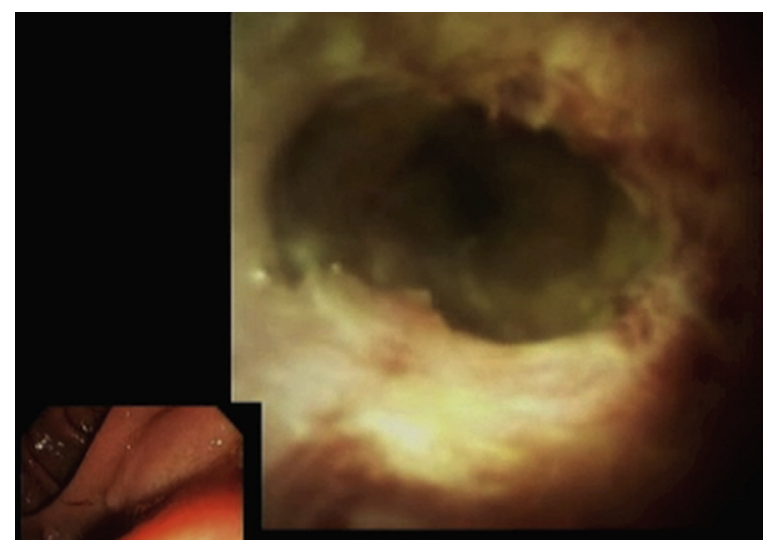

Figure 6. Six-month surveillance view showing scarring at treatment site without residual polyp.

followed by 2 additional polypectomies for residual tissue, and intraductal balloon dilation with APC ablation (3 ablations, with 2 performed concurrently with polypectomy). The procedures were performed at intervals of 2 to 3 months. Although endoscopically there appeared to be resolution of disease, biopsy specimens from the distal CBD showed that tubulovillous adenoma without dysplasia persisted (Figs. 2 and 3). The decision was made to trial RFA.

The extent of intraductal involvement was clearly visualized with per-oral cholangioscopy. Adenomatous tissue was seen in the distal portion of the CBD extending circumferentially (Fig. 4). An $8 \mathrm{~F}$ probe with a $2.5-\mathrm{cm}$ ablation zone (Fig. 5) was positioned for RFA (settings of effect 8 and 7 watts) at the distal biliary tree. Positioning was determined by aligning the area of biliary ablation between the ring electrodes located on the catheter ablation zone. After a 90-second ablation, eschar was seen at the biliary orifice. Cholangioscopy showed a significant treatment effect, with thermal injury to the adenomatous tissue and biliary wall. At a 3-month surveillance visit, the patient was treated with a second RFA session (settings of effect 8 and 10 watts for 90 seconds) for small residual polyp tissue. Ten French $\times 7 \mathrm{~cm}$ plastic stents were placed prophylactically into the CBD after each session. The patient was without endoscopic and histologic evidence of recurrence or adverse events at a 6-month surveillance visit (Fig. 6).

Our experience suggests that RFA treatment for intraductal extension of ampullary adenomas can be an effective option when prior techniques have proved unsuccessful. The use of cholangioscopy was essential to evaluate the extent of intraductal tissue. Prophylactic stent placement should be considered for the CBD and PD to help prevent stricture formation and post-ERCP pancreatitis as described in previous series.

\section{DISCLOSURE}

All authors disclosed no financial relationships relevant to this publication. 


\section{ACKNOWLEDGMENT}

The authors would like to thank Aslam Syed, MD, for assisting in finalizing and submitting the manuscript.

Abbreviations: APC, argon plasma coagulation; $C B D$, common bile duct; $P D$, pancreatic duct; RFA, radiofrequency ablation.

\section{REFERENCES}

1. Rustagi $T$, Irani S, Reddy D, et al. Radiofrequency ablation for intraductal extension of ampullary neoplasms. Gastrointest Endosc 2017;86:170-6.
2. Suarez AL, Coté GA, Elmunzer BJ. Adjunctive radiofrequency ablation for the endoscopic treatment of ampullary lesions with intraductal extension (with video). Endosc Int Open 2016;4:E748-51.

3. Valente $R$, Urban $O$, Del chiaro $M$, et al. ERCP-directed radiofrequency ablation of ampullary adenomas: a knife-sparing alternative in patients unfit for surgery. Endoscopy 2015;47:E515-6.

Department of Gastroenterology, Allegheny Health Network, Pittsburgh, Pennsylvania, USA.

Copyright (c) 2018 American Society for Gastrointestinal Endoscopy. Published by Elsevier Inc. This is an open access article under the CC BYNC-ND license (http://creativecommons.org/licenses/by-nc-nd/4.0/).

http://dx.doi.org/10.1016/j.vgie.2017.12.002 